\title{
PERFORMANCE IMPROVEMENT OF ENERGY AWARE AND ADAPTIVE ROUTING PROTOCOLS FOR MANETS - A SURVEY
}

\author{
Deepthy $\mathbf{J}^{1}$, Nishanth Krishnan ${ }^{2}$ \\ ${ }^{I}$ PG Student, [Wireless Technology], ${ }^{2}$ Assistant Professor, Dept. of ECE, Toc H Institute of Science and Technology, \\ Kerala, India
}

\begin{abstract}
Mobile Ad hoc Networks are infrastructure-less networks where each node acts as sender, receiver as well as router for exchange of data. These nodes are usually battery-powered and often they die out before data transmission is complete. These networks are highly dynamic due to node mobility leading to frequent link breaks. This gives rise to added route repairs which increase routing overhead and use up battery resource of nodes unnecessarily. As a result, lifetime of the network is considerably reduced. Hence, the main target of routing protocols is to create an energy aware and adaptive network in order to prolong its lifetime. Interference from other mobile hosts is also a major limitation that degrades protocol performance. When protocol enhancements are proposed, reactive routing protocols such as AODV and DSR are given prime importance because of their on-demand characteristics. Therefore, the highlight is on reducing interference and energy consumption which could ensure availability of discovered path for the complete duration of information transfer. It is observed from research studies that incorporating a cross layer approach can further improve performance metrics such as throughput, end-to-end delay and control overhead of the network. This paper emphasizes on the study of different energy aware and adaptive modifications of routing protocols and the proposed solutions to achieve performance gain in MANET.
\end{abstract}

Keywords: MANETs, Route repairs, Energy aware Adaptive routing, Cross layer, Interference

\section{INTRODUCTION}

A Mobile Ad-hoc Network (MANET) can be defined as a network consisting of autonomous and self-organizing wireless nodes without any aid of a centralized base station administration or infrastructure. The nodes themselves are transmitters, receivers and routers. In order to route packets or data to an intended destination, a routing protocol becomes necessary. The two main classes of routing protocols are Proactive routing protocols (also called as Table Driven protocols) and Reactive (also called On-demand protocols) routing protocols. Proactive protocols keep track of all the topology changes in the network by periodically sending route advertisements thus establishing routes to all the possible destinations in the network. The main drawback of this protocol is that there is huge wastage of resources as routes to unwanted destinations are also updated and stored in routing tables. Examples of proactive protocols are Destination Sequenced Distance Vector (DSDV) protocol, Optimum Link State Routing (OLSR) protocol etc. However, reactive routing protocols determine route only if a node has a packet to send to a particular destination. This saves bandwidth as there are no periodic route advertisements and updating of route tables. But the disadvantage here is that, initial latency would be slightly high compared to proactive protocols. Examples of reactive protocols are Ad hoc On demand Distance Vector (AODV) routing, Dynamic Source Routing (DSR) etc.
Each node within a MANET is characterized by its mobility. It is free to move in any random direction with any arbitrary speed. But this leads to frequent topology changes which in turn results in link breaks. When link failure increases, it becomes much difficult to find out routes that could persist for the transfer of entire data. Moreover, energy is used up for additional route discovery and route maintenance which would degrade the network lifetime. Hence, our focus is to make the nodes aware of its own energy while participating in routing. Conventional routing protocols establish an alternate route only after a link has actually failed. Here, we highlight the importance of an adaptive network to reduce link breaks by a make-before-break mechanism. This could help to proactively establish an alternate route much before the actual link break thus ensuring a fully connected network at all times. We also consider the effect of interference from other mobile nodes on the performance of protocol.

A full cross layer design, introduces stack wide layer interdependencies to optimize overall network performance. In cross layering, protocols use the state information flowing throughout the stack to adapt their behavior accordingly [1]. Information can be shared between layers which are nonadjacent unlike in traditional layered architecture. This leads to faster performance gains and QoS improvements. For example, the physical layer information such as power, speed, noise to be used according to a specific application. Cross 
layer approach introduces the possibility that inside the layered architecture, the protocols belonging to different layers can cooperate by sharing network-status information while still maintaining separation between the layers in protocol design.

The literature survey [1-16] shows the modifications that have been done with major routing protocols in order to achieve the performance gain. Also, it shows the enhancement in performance metrics when a cross layer solution is used. The following section depicts the studies carried out to achieve performance improvement for energy aware and adaptive routing protocols for MANETs.

\section{RELATED WORK}

In order to make the routing energy aware and adaptive to network condition, a number of solutions have been proposed in the literature survey. It has been shown in [2] that, the proposed protocol "EBL (A Routing Protocol for Extend network life time through the Residual Battery and Link Stability in MANET) could minimize energy consumption and allow balanced energy consumption in MANET by considering parameters- residual battery capacity, link stability and distance between neighbor nodes.. EBL sets up threshold for these parameters while broadcasting route request (RREQ) message to its neighbors. Intermediate node compares its own battery capacity, link stability and hop distance to its neighbor with the threshold to determine whether this intermediate node could participate in routing. Highest priority is assigned to residual battery capacity and the least for distance among neighbor nodes. The performance of EBL is found to be better than those protocols that use only hop count as a metric. But the protocol doesn't perform well with little or no mobility. Signal Stability Adaptive Routing (SSA) classifies the links into strong and weak depending on the received signal strength measured when packets are forwarded through them [3]. Stable links ensure longer lived routes and less route maintenance. When a node receives a RREQ packet, it processes the request only if it is received by a strong link, otherwise the packet is dropped. However, when a failed link is detected, an intermediate node sends an error message to the source node to notify it that the path is broken. Then the source reinitiates another route discovery which causes undue overhead and is thus undesirable.

In [4], the authors consider the impact of link stability, remaining energy and delay in forwarding packets between nodes in making routing stable and energy aware. When a RREQ packet arrives at the destination at first, the sum of delay of intermediate nodes should be minimized and nodes with high delay nodes should avoid joining the route [4]. An intermediate node is selected on the basis of its remaining battery capacity, stability and also the delay incurred while it forwards control packets. The protocol when simulated has shown improvement with respect to packet delivery, end-toend delay etc. compared to AOMDV and other protocols.

Morteza Maleki et.al. in their paper Power-aware Source Routing (PSR) [5] provides a modification to DSR protocol by taking into account both node mobility and energy depletion of the nodes as the reason for link failure in MANET. Intermediate nodes use a battery cost function of routes which is updated in header of RREQ. These nodes select the route with minimum battery cost. Thus this protocol ensures that no path would be overused and each selected path has minimum battery cost among all possible path between two nodes. However, the route discovery and maintenance are much complicated compared to DSR as PSR considers both mobility and energy depletion scenarios for path invalidity.

In [6], authors propose a cross-layer approach to select a stable route, based on node's current energy. They considered parameters such as current energy of node, its average energy drain-rate and specified session-duration as the criterion for selecting an intermediate node. They also propose a "makebefore-break" mechanism for finding an alternate route for the session, when the node's energy gets drained before the session is complete. The uniqueness of this improved version of AODV is that as it uses only local information, there is no additional communication overhead.

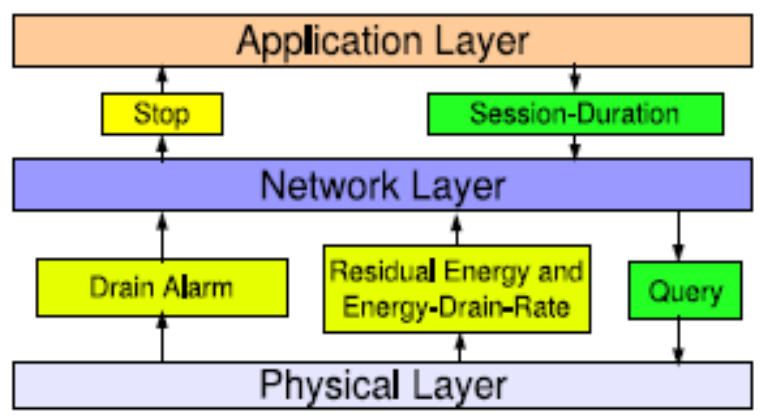

Fig - 1: Cross layer architecture in SQ-AODV [6]

Due to the selection of energy aware node, it is possible to obtain substantial improvement in QoS in terms of packet delivery ratio and packet delay. Also, the adaptive makebefore-break mechanism, SQ-AODV proactively reroutes sessions thus avoiding packet drop. This again results in nearzero packet loss and superior QoS performance.

Arun Avudainayagam, Wenjing Lou, and Yuguang Fang, in their paper "DEAR: A Device and Energy Aware Routing protocol for heterogeneous ad hoc networks" consider two different classes of nodes, battery-powered nodes and externally powered nodes [7]. By embedding both the energy and the device-type awareness into the routing protocol, they force the externally powered nodes to forward more traffic and perform more routing functions than a battery-powered node. They incorporate a redirect scheme in DEAR that actively 
redirects the packets to the powered nodes for power-saving operations. They also assume that an externally powered node has the capability to increase its transmission power to a higher level so that it can reach any other node in the network in one hop. The real advantage of DEAR is not apparent in the static case.

In [8], the authors incorporate a cross layer approach by utilizing stability of routes as the criterion for selecting a route among the multiple discovered paths. In this sense, the received signal strength is considered an indirect measurement of a route lifetime. The protocol reduces the number of route discoveries on the network as it selects stable routes. However, the exclusive utilization of the signal strength information without considering the number of hops does not guarantee any improvement on the network performance. Also when the traffic increases, the interferences increase and then, a bigger amount of packets are lost.

Signal Strength and Energy aware Dynamic Source Routing (SEA-DSR) directly incorporates signal strength and residual battery capacity into route selection criteria through a cross layer approach [9]. The authors use a reliability count factor which is a measure of the signal strength at which an intermediate node receives RREQ plus the residual battery energy of the node. Destination node selects the most reliable route among all the routes based on this value and the hop count value.

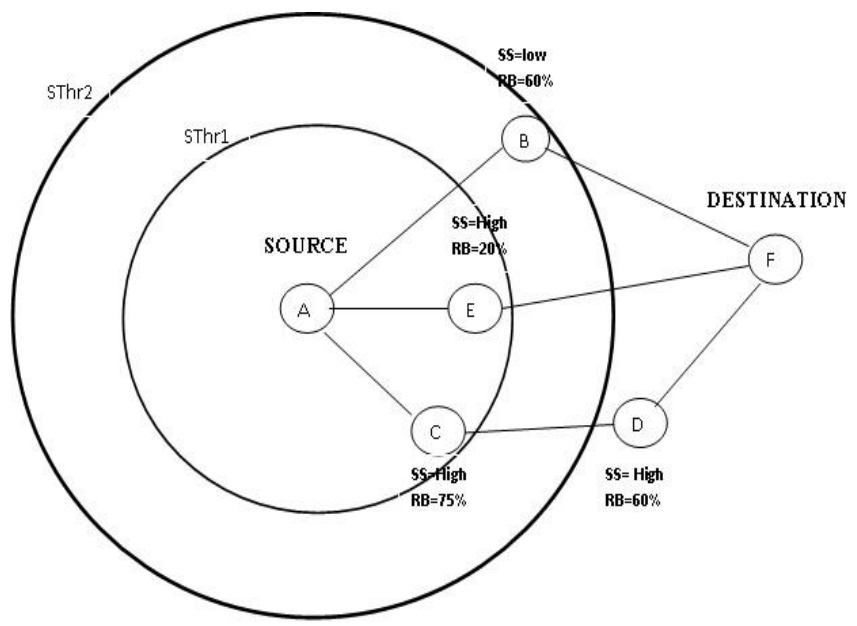

Fig - 2: Route Establishment of SEA-DSR [9]

Referring to Figure 2, suppose node A wants to communicate with node $\mathrm{F}$, for which it does not have a route, node $\mathrm{A}$ broadcasts a RREQ packet to its neighbors. The intermediate nodes "B", "C", "D" and "E" on receiving the RREQ packet, updates the reliability count field in it based on the strength of the packet it received and the residual energy of the node. Then it rebroadcasts the RREQ packet to the neighbor nodes. The destination node " $F$ " on receiving its first RREQ, starts a timer for $\Delta \mathrm{t}$ sec. After the timer expires, it selects the path with the highest reliability count. RREP is sent in that path. The reliability factor for the received path is calculated by the following formula [9]:

Reliability Factor $=\frac{\text { Reliability count }}{\text { No. of Hops }}$

Source node A on receiving the RREP packet starts data transmission on that path. The protocol also incorporates an adaptive make-before-break strategy to reduce routing overhead by going for local route recovery. However, the protocol exhibits a slightly high delay in case of low mobility. Also, the performance could degrade when the traffic is high in a less mobile scenario.

Route Discovery by Cross Layer Routing Protocol (RDCLRP) is introduced by the authors keeping in their mind, the following objectives [10] :

- Minimize delay in route searching

- Minimize communication overhead

- Minimize battery power consumption

- Local repair method

- Maximize delivery rate

- $\quad$ Node mobility

RDCLRP makes use of adaptive Hello Warning Messages (HWM) to warn the nodes before a link break. This is achieved by monitoring the position and battery power of the nodes, thus a cross layer approach is used to share these parameters from physical layer to network layer. Using fuzzy rules, a Hello Interval (HI) is set up during which HWM messages are sent by critical nodes. High traffic is a major hindrance which could degrade RDCLRP's performance.

P. Srinivasan and P. Kamalakkannan address the significance of route maintenance and restoration factor in designing routing protocols in their work "Enhancing Route Maintenance in RSEA-AODV for Mobile Ad Hoc Networks". They propose a new method to enhance the route maintenance process in Route Stability and Energy Aware Routing (RSEAAODV) protocol that takes into account both stability and energy metrics during route discovery, through cross layer approach [11]. The parameters cross layered from physical to network layer are signal strength of the node at which it received the packet, residual energy of node and interface queue length at periodic intervals. This protocol also goes for an adaptive make-before-break route repair mechanism which reduced the routing overhead and reduced the packet drops.

It can be seen from the survey that the main issues in routing data in MANET are the energy constrained nodes, limited bandwidth and the node mobility. In addition to that, from the survey it is observed that when traffic increases, interference from other mobile hosts is a major hindrance which reduces the performance of the protocol. Many studies have been 
carried out to improve the conventional routing protocols to make them adaptive to network changes and energy efficient. Cross layer approach is an emerging paradigm that allows sharing of information between various non-adjacent layers of OSI communication model. It helps to achieve high data rates and higher performance gains. So the main goal of this paper is to propose a cross layer routing protocol which is energy aware and adaptive that takes into account, high traffic scenario where nodes are affected by interference from other mobile nodes thus increasing packet drops. This is obtained by making necessary changes to the popular AODV routing protocol.

\section{PROPOSED SOLUTION}

When modifications are executed in AODV, performance of the protocol increases manifold. Interference from other hosts under high traffic situations could be reduced by introducing a parameter called Signal-to-Interference-and-Noise-Ratio (SINR) at physical layer and cross layering it to network layer. At network layer, it is compared with SINR threshold [12]. Only those packets which have a higher value of SINR than the SINR threshold are considered. This makes the processing of messages by AODV selective, thus discarding noisy signals. Energy awareness and adaptivity could be achieved through the proper selection of intermediate nodes during the routing of data.

The proposed solution is obtained by incorporating the parameters- signal strength/SINR, residual battery capacity and interface queue length from physical layer to network layer by cross layering. When traffic is low, parameter to be used is signal strength and under high traffic case, instead of signal strength, SINR parameter is to be used. Interface queue is the queue between MAC layer and LLC (Logical Link Control) layer. This parameter is used to detect link breaks due to congestion in the network.

A similar approach is used in [9] to make DSR energy aware and adaptive. However, there is an inherent drawback for DSR that it uses source routing where the entire route is a part of the header. Therefore, packet header grows in size when additional fields are included. This may degrade the performance of the network even more. Hence, enhancing AODV is a much better solution compared to DSR.

\subsection{Route Discovery Phase}

Threshold values for signal strength/SINR, residual battery capacity are set up and included in the field of RREQ packet before broadcasting. When an intermediate node receives the RREQ packet, it invokes information about its remaining energy and signal strength/SINR from the physical layer. This information is used in network layer for routing (cross layer). Node compares its values with the threshold value and will process the RREQ only if their values are above the threshold.
Otherwise the RREQ is dropped. This ensures that the route is energy efficient and stable for the data transfer.

\subsection{Route Maintenance Phase}

Threshold values are set up for signal strength and residual battery capacity and included with RREP packet. An intermediate node on receiving RREP gets information about these parameters from physical layer. These values are compared with the threshold to detect whether that node's energy and stability are enough to last for the data transmission. If not, then the node sends an ALERT packet to all its 1 hop neighbors for an alternate path. Thus a new path could be established much before the actual link failure.

\section{CONCLUSIONS}

Considering energy conservation and network connectivity issues, many protocols have been modified to obtain the desired results. The major task of routing protocol is to make the nodes aware of the network conditions and its own energy before using an established path. Hence, there arises the need for an energy aware and adaptive routing protocol. Also, when designing an efficient routing protocol, noise and interference cannot be neglected. The proposed solution could achieve these objectives by making necessary changes to AODV through a cross layer approach. It introduces SINR parameter which takes care of reducing interference from other nodes in a high traffic condition. To obtain energy efficient routing, signal strength, residual battery capacity and queue length are monitored before processing a packet. Also, an adaptive make-before-break strategy is adopted for early detection of link failures, to prolong the network lifetime. Simulation studies are to be performed to achieve the expected improvements regarding throughput, end-to-end delay, routing overhead and packet delivery ratio using NS2.

\section{ACKNOWLEDGEMENTS}

The ongoing work based on the suggested solutions could not have been possible without Mr. George M. Jacob, Lab Instructor, Toc $\mathrm{H}$ Institute of Science and Technology who have encouraged us throughout to address the problem. We thank him for his constant help and support.

\section{REFERENCES}

[1]. Marco Conti, Gaia Maselli, Giovanni Turi, "CrossLayering in Mobile Ad Hoc Network Design", IEEE Computer Society, February 2004, pp : 48-51.

[2].GunWoo Park and SangHoon Lee, "A Routing Protocol for Extend Network Lifetime through the Residual Battery and Link Stability in MANET",Applied Computing Conference (ACC '08), Istanbul, Turkey, May 27-30, 2008, pp : 199-204.

[3]. Rohit Dube, Cynthia D Rais, Kuang-Yeh Wang and Satish K Tripathi, "Signal Stability- based Adaptive Routing (SSA) 
for Ad hoc Mobile Networks, IEEE Personal Communications, Volume- 4, February 1997, pp: 36-45.

[4]. Senthil Murugan Tamilarasan and Kannan Eswariah, "Link Stability With Energy Aware Ad Hoc On Demand Multipath Routing Protocol In Mobile Ad Hoc Networks" ,American Journal of Applied Sciences 10 (8)., July 10, 2013, pp: 844-848.

[5]. Morteza Maleki, Karthik Dantu, and Massoud Pedram, "Power-aware Source Routing Protocol for Mobile Ad Hoc Networks", IEEE proceedings on Low power electronics and design, Monterey, California USA, August 12-14 2002, pp: 72-75.

[6]. Mallapur Veerayya, Vishal Sharma, Abhay Karandikar, "SQ-AODV: A Novel Energy-Aware Stability-Based Routing Protocol for Enhanced Qos In Wireless Ad-Hoc Networks", IEEE Military Conferences, San Diego, USA, November 1619, 2008, pp:1-7.

[7]. Arun Avudainayagam, Wenjing Lou, and Yuguang Fang, "DEAR: A Device and Energy Aware Routing protocol for heterogeneous ad hoc networks", IEEE proceedings on MILCOM, October 7-10, 2002, Volume- 1, pp: 483 - 488.

[8]. Alicia Triviño-Cabrera, Irene Nieves-Pérez, Eduardo Casilari, Francisco J. González-Cañete, "Ad Hoc Routing Based on the Stability of Routes" Dpto. Tecnología Electrónica, University of Málaga ETSI Telecomunicación, Malaga Spain, Campus de TeatinosMobiWAC'06, October 2, 2006, pp : 6-10.

[9]. P.Srinivasan, K.Kamalakkannan, "Signal Strength and Energy aware Reliable Route Discovery in Manet" International Journal of Communication Network Security ISSN: 2231- 1882, Volume-1, Issue-4, 2012, pp: 55-59.

[10]. Mehajabeen Fatima, Roopam Gupta, T.K. Bandhopadhyay, "RDCLRP- Route Discovery by Cross Layer Routing Protocol for Performance Improvement", IEEE International Conference, Nagpur Maharashtra, India, April 21-22, 2011, pp : 300-303.

[11]. P.Srinivasan, K.Kamalakkannan, "Enhancing Route Maintenance in RSEA-AODV for Mobile Ad hoc Networks", Proceedings of IEEE 7th International Conference on Intelligent Systems and Control (ISCO), Coimbatore, Tamil Nadu India, January 4-5, 2013, pp : 464-469.

[12]. Rekha Patil, Vijay.K.Kerji, "SINR Based Routing for Mobile Ad hoc Networks", 3rd International Conference on Electronics Computer Technology (ICECT), Volume- 5, Kanyakumari India, April 8-10, 2011, pp:369-372.

[13]. Marco Di Felice, "Cross-Layer Optimizations in MultiHop Ad Hoc Networks", University of Bologna, Padova Italy, March 2008.

[14]. Charles.E.Perkins, Elizabeth.M.Royer, "Ad-hoc On Demand Distance Vector Routing", 2nd IEEE Proceedings on Mobile Computing Systems and Applications, New Orleans, USA, February 25-26, 1999.

[15]. Dr.A.Ramesh Babu, Prof.S.Meenakshi Sundaram, "Performance Optimization of Routing Protocols in Mobile Ad hoc Networks: Issues and Challenges", International
Journal of Computational Engineering Research, Volume-3, Issue-5, May 2013, pp :84-89.

[16]. Byoungheon Shin, Yangwoo Ko, Seonyeong Han, Dongman Lee, "SINR-Based Link-Quality Routing Protocol in Multi-Radio Mobile Ad-Hoc Networks" AsiaFI'10 Summer School, Yokohama, Japan, August 24-26, 2010.

\section{BIOGRAPHIES}

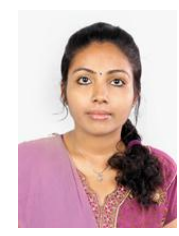

Deepthy J received her B.Tech. Degree in Electronics and Communication from Toc $\mathrm{H}$ Institute of Science and Technology, Kerala in 2012. Currently she is pursuing her M.Tech. degree in Wireless Technology at Toc H Institute of Science and Technology. Her areas of interests include ad-hoc \& sensor networks and mobile communication.

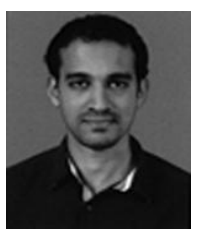

Nishanth Krishnan received his B.Tech. in Electronics and Communication from NSS College of Engineering, Palakkad, Kerala in 2006 and M.Tech. from Sona College of Technology, Salem, Tamil Nadu in 2008. He is currently associated with Toc $\mathrm{H}$ Institute of Science and Technology, Kerala as an Assistant Professor in the department of Electronics and Communication since 2009. His areas of interests include Computer Communication \& Networking and Digital Image Processing. 